\title{
Risk factors for progression of ossification of the posterior longitudinal ligament in asymptomatic subjects
}

\author{
Toru Doi, MD, PhD, ${ }^{1}$ Ryuji Sakamoto, MD,1 Chiaki Horii, MD,1 Naoki Okamoto, MD,1 \\ Koji Nakajima, MD, ${ }^{1}$ Shima Hirai, MD, ${ }^{1}$ Fumihiko Oguchi, MD, ${ }^{1}$ So Kato, MD, PhD, ${ }^{1}$ \\ Yuki Taniguchi, MD, PhD, ${ }^{1}$ Yoshitaka Matsubayashi, MD, ${ }^{1}$ Naoto Hayashi, MD, PhD, ${ }^{2}$ \\ Sakae Tanaka, MD, PhD, ${ }^{1}$ and Yasushi Oshima, MD, PhD ${ }^{1}$
}

\begin{abstract}
1Department of Orthopaedic Surgery, University of Tokyo; and 'Department of Computational Diagnostic Radiology and Preventive Medicine, The University of Tokyo Hospital, Tokyo, Japan
\end{abstract}

\begin{abstract}
OBJECTIVE The incidence and risk factors for the progression of ossification of the posterior longitudinal ligament (OPLL) have been previously reported in surgically and nonsurgically treated symptomatic patients. However, the correlates of OPLL progression in asymptomatic subjects with OPLL are not well characterized. This study aimed to clarify the incidence and risk factors for OPLL progression in asymptomatic subjects based on whole-body CT.

METHODS The authors retrospectively reviewed 2585 healthy subjects who underwent whole-body CT at a single health center from September 2007 to December 2011. This study included asymptomatic subjects with OPLL who underwent CT scans twice with an interval of at least 5 years. Progression of OPLL was assessed based on initial and final CT scan. Subjects were divided into two groups: nonprogression (OPLL-NP) and progression (OPLL-P) groups. Clinical characteristics, bone mineral density status, OPLL types, and OPLL involvement of multiple vertebral levels between the two groups were compared. Risk factors for progression of OPLL were identified by logistic regression analysis after propensity score adjustment.
\end{abstract}

RESULTS Of the 109 subjects with OPLL (91 men and 18 women), 20 (18.3\%) exhibited OPLL progression (OPLL-P group). Subjects in the OPLL-P group were significantly younger $(p=0.031)$, had higher prevalence of multilevel OPLL involvement $(p=0.041)$ and continuous type of OPLL $(p=0.015)$, and had higher uric acid (UA) levels $(p=0.004)$ at the time of initial CT examination compared to the OPLL-NP group. Younger age (adjusted odds ratio [aOR] 0.95, 95\% $\mathrm{Cl} 0.90-$ 0.99), OPLL involvement of multiple vertebral levels (aOR 2.88, 95\% CI 1.06-7.83), continuous type of OPLL (aOR 4.21, $95 \% \mathrm{Cl} 1.35-13.10$ ), and higher UA levels (aOR 2.09, 95\% Cl 1.24-3.53) were significant risk factors for OPLL progression.

CONCLUSIONS Younger age, OPLL involvement of multiple vertebral levels, continuous type of OPLL, and higher UA levels are significant risk factors for OPLL progression in asymptomatic subjects.

https://thejns.org/doi/abs/10.3171/2020.3.SPINE2082

KEYWORDS asymptomatic subjects; continuous type; hyperuricemia; ossification of the posterior longitudinal ligament; OPLL; progression; risk factors; degenerative

$\mathrm{O}$ SSIFICATION of the posterior longitudinal ligament (OPLL) refers to abnormal ectopic ossification of the posterior longitudinal ligament. OPLL is reportedly more common in males, elderly people, and in the Asian population., ${ }^{1,2}$ The cervical spine is most commonly affected by OPLL. ${ }^{3,4}$ OPLL may occasionally cause neurological deficit due to compression of the spinal cord by an ossified ligament. ${ }^{3,4}$ Patients with severe neurological dysfunction require surgical treatment. ${ }^{5}$ OPLL is also incidentally detected in adults during CT for suspected trauma or cancer screening. ${ }^{1,6}$ There is no clear consensus on the management of OPLL in asymptomatic patients or in those with mild symptoms.?

An OPLL mass may show progression both in the postoperative course ${ }^{8-15}$ and in the natural course ${ }^{16-21}$ resulting in severe spinal cord compression. Several risk factors such as younger age, continuous or mixed type morphology, greater segmental motion, and involvement of multiple

ABBREVIATIONS aOR = adjusted odds ratio; BMD = bone mineral density; $\mathrm{BUA}=$ broadband ultrasound attenuation; HbA1c = glycosylated hemoglobin; $\mathrm{HDL}=$ highdensity lipoprotein; LDL = low-density lipoprotein; OPLL = ossification of the posterior longitudinal ligament; OPLL-NP = OPLL nonprogression; OPLL-P = OPLL progression; QUS = quantitative ultrasonography; SOS = speed of sound; TC = total cholesterol; TG = triglyceride; UA = uric acid.

SUBMITTED January 18, 2020. ACCEPTED March 6, 2020.

INCLUDE WHEN CITING Published online May 8, 2020; DOI: 10.3171/2020.3.SPINE2082. 
levels were found to be associated with OPLL progression after surgical intervention. ${ }^{8-12,14,22}$ In the context of natural disease course, young age, C2-3 involvement, and mixed type of OPLL are some of the reported risk factors for OPLL progression in nonsurgically treated patients. ${ }^{16,19}$

The abovementioned previous studies did not include asymptomatic patients with OPLL. Characterization of OPLL progression in the natural course may help improve the management of asymptomatic subjects with incidentally detected OPLL; however, the incidence or risk factors for OPLL progression in asymptomatic subjects are not well characterized.

In the present study, we aimed to analyze the natural course of OPLL mass in asymptomatic subjects who underwent whole-body CT scans as part of their routine medical checkup at baseline and more than 5 years after the initial CT scan. The objective was to clarify the incidence and risk factors for the progression of the ossified mass in asymptomatic subjects with OPLL.

\section{Methods \\ Study Subjects}

The study was approved by the IRB of the Clinical Research Support Center of the University of Tokyo Hospital. Written informed consent was obtained from all subjects. The subjects in this study comprised healthy individuals who had undergone whole-body CT scans for cancer screening at our institution between September 2007 and December 2011. All CT volumes were scanned by a GE Light Speed Scanner (GE Healthcare Japan; $120 \mathrm{kV}, 80 \mathrm{~mA}, 512 \times 512$ matrix). We retrospectively reviewed axial CT images of 2585 subjects in the bone window with automatic adjustment. The diagnosis of OPLL was established by two experienced spine surgeons (T.D. and S.H.). OPLL was defined as ossification of the posterior longitudinal ligament with more than a $2-\mathrm{mm}$ thickness $^{6,23}$ from the atlas to the pelvis. Among subjects who were diagnosed with OPLL, we selected those who underwent follow-up CT at least 5 years after the initial examination. Subjects who underwent surgical treatment for OPLL during the follow-up period were excluded because this study focused on the natural course of OPLL mass in asymptomatic subjects.

\section{Measurements of the Ossified Lesion and the Definition of OPLL Progression}

We measured the maximum anteroposterior thickness of the ossified lesion ( $\mathrm{mm}$ ) and anteroposterior diameter of the spinal canal ( $\mathrm{mm}$ ) in the sagittal view of reconstructed CT images. The maximum occupancy ratio of the OPLL mass was calculated (percentage: anteroposterior thickness of OPLL/anteroposterior diameter of spinal canal). We also measured the longest distance between the proximal and distal margins of the OPLL mass on the sagittal view (mm). OPLL measurements were obtained at the initial and final CT examinations by two spine surgeons (T.D. and R.S.). The measured values were expressed as the average of measurements by two surgeons. Axial and sagittal CT images were reconstructed using the RadiAnt DICOM Viewer software (Medixant Co., Ltd.) and were observed in the bone window with automatic adjustment. OPLL progression was defined as an increase of more than 2-mm in the sagittal thickness and/or the longitudinal distance of ossified mass, which is the most commonly used criteria of OPLL progression..$^{8-10,12}$ Based on these findings, the subjects were classified into two groups: nonprogression (OPLL-NP) and progression (OPLL-P) groups. The OPLL was categorized into four types (continuous, segmental, mixed, and localized) according to the Investigation of Public Health and Welfare's classification. ${ }^{23}$ We investigated the spinal region (cervical, thoracic, or lumbar) of maximum OPLL mass (maximum sagittal thickness or longitudinal distance). We also assessed OPLL involvement at multiple vertebral levels, which was defined as an ossified lesion in more than 3 vertebral levels in the sagittal view.

\section{Data Collection}

We examined the subjects' characteristics and clinical data, including age, BMI at the time of initial CT examination, and the following laboratory data at the time of initial and final CT examinations: glycosylated hemoglobin level (HbA1c), serum triglyceride (TG), total cholesterol (TC), high-density lipoprotein (HDL) cholesterol, low-density lipoprotein (LDL) cholesterol, uric acid (UA), calcium, and phosphorus. Bone mineral density (BMD) status of the calcaneus at the time of initial and final CT examinations was examined for screening of osteoporosis using quantitative ultrasonography (QUS); QUS is widely utilized for assessment of BMD status. QUS parameters include broadband ultrasound attenuation (BUA), a measure of the frequency dependence of ultrasound attenuation, and speed of sound (SOS), which reflects the transmission velocity of ultrasound passing through a soft tissue. QUS measurements were obtained using an A-1000 EXP II machine (GE Healthcare Japan). We examined the subjects with suspected cancer based on the radiography interpretation report of the initial CT examination. Among the subjects with suspected cancer, we identified those with a definitive diagnosis for cancer by pathological findings in biopsies and investigated subsequent treatment for cancer.

\section{Statistical Analysis}

Cohen's kappa coefficient $(\kappa)$ values were calculated to assess interrater reliability for the judgment of OPLL progression. Interrater reliability was classified as poor when the $\kappa$ value was $\leq 0.20$, fair when it was $0.21-0.40$, moderate when $0.41-0.60$, good when $0.61-0.80$, and excellent when $>0.80 .{ }^{29}$ Data are expressed as mean (standard deviation [SD]; range) or mean (SD). Continuous data were compared using the unpaired t-test, while categorical data were compared using Fisher's exact test. Multivariate logistic regression analysis was performed to identify factors associated with OPLL progression. Variables associated with $\mathrm{p}$ values $<0.05$ on univariate analysis were subjected to multivariate analysis as candidate risk factors (age, OPLL involving $\geq 3$ vertebrae, continuous type of OPLL, UA). Due to the relatively low number of subjects with OPLL progression, propensity score adjustment was used for each of the candidate risk factors in the multivari- 


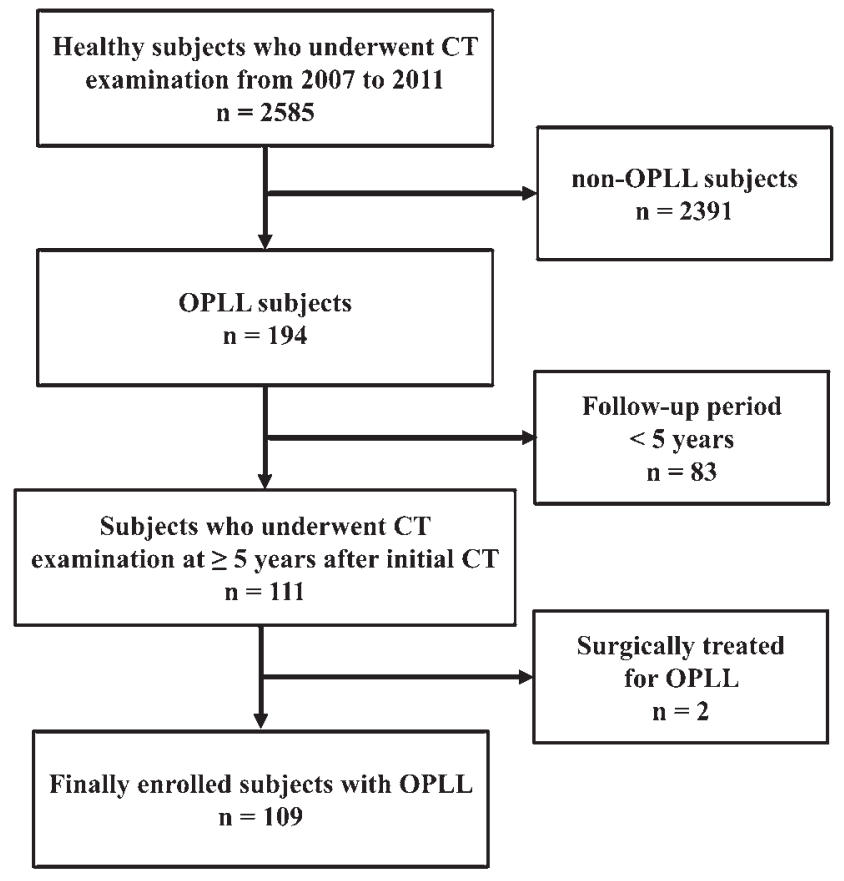

FIG. 1. Flowchart of the study population.

ate analysis. Propensity score adjustment preserved the statistical power by reducing covariates to a single variate. A propensity score was created using binary logistic regression to predict the progression of OPLL as a function of basic factors such as sex and BMI. All data were analyzed using IBM SPSS Statistics (version 23, IBM Corp). A p value $<0.05$ was considered indicative of a statistically significant difference.

\section{Results \\ Study Subjects}

The mean age of all subjects $(\mathrm{n}=2585)$ was $57.9 \pm 11.0$ years old (range 23-90 years) and the BMI was $23.8 \pm$ $3.5 \mathrm{~kg} / \mathrm{m}^{2}$ (range $12.9-42.3 \mathrm{~kg} / \mathrm{m}^{2}$ ); males accounted for $68.7 \%(\mathrm{n}=1775)$ of the study population. Among the 2585 subjects, 194 subjects (7.5\%; 159 men and 35 women) were diagnosed with OPLL in the whole spine based on the axial CT images. Of these subjects, we selected 111 who underwent follow-up CT at least 5 years after the initial examination. Two subjects who underwent surgical treatment for OPLL (a 67-year-old woman who underwent anterior decompression and fusion surgery, and a 55-year-old man who underwent laminoplasty) within 1 year after initial CT examination were excluded. Finally, 109 asymptomatic subjects with OPLL were investigated for OPLL progression in this study (Fig. 1).

\section{Demographic Data}

The mean age of the 109 subjects with OPLL (91 men and 18 women) was $61.6 \pm 10.4$ years old (range 40-85 years) and the average BMI was $25.1 \pm 3.1 \mathrm{~kg} / \mathrm{m}^{2}$ (range $17.8-33.3 \mathrm{~kg} / \mathrm{m}^{2}$ ). In 42 subjects (38.5\%), OPLL involved $\geq 3$ vertebral levels. The distribution of the various types
TABLE 1. Demographic data of 109 subjects with OPLL

\begin{tabular}{lc}
\hline \multicolumn{1}{c}{ Variable } & \multicolumn{1}{c}{ Value } \\
\hline Males, $\mathrm{n}(\%)$ & $91(83.5)$ \\
\hline Mean age (SD), yrs & $61.6(10.4)$ \\
\hline Mean BMI (SD), $\mathrm{kg} / \mathrm{m}^{2}$ & $25.1(3.1)$ \\
\hline Mean follow-up period of CT scans (SD), mos & $80.8(15.5)$ \\
\hline OPLL $\geq 3$ vertebral levels, $\mathrm{n}(\%)$ & $42(38.5)$ \\
\hline Type of OPLL, $\mathrm{n}(\%)$ & $17(15.6)$ \\
\hline Continuous & $82(75.2)$ \\
\hline Segmental & $6(5.5)$ \\
\hline Mixed & $4(3.7)$ \\
\hline Localized & $106(97.2)$ \\
\hline Spinal region of maximum OPLL mass, $\mathrm{n}(\%)$ & $3(2.8)$ \\
\hline Cervical & $0(0.0)$ \\
\hline Thoracic & \\
\hline Lumbar & $3.4(1.0)$ \\
\hline OPLL size at initial examination (SD) & $28.8(8.8)$ \\
\hline Mean thickness of maximum region of OPLL, mm & $18.1(12.5)$ \\
\hline Occupancy ratio of OPLL, \% & $3.8(1.1)^{*}$ \\
\hline Mean length of maximum region of OPLL, mm & $31.4(9.8)^{*}$ \\
\hline OPLL size at final examination (SD) & $20.2(15.2)$ \\
\hline Mean thickness of maximum region of OPLL, mm & \\
\hline Occupancy ratio of OPLL, \% & \\
\hline Mean length of maximum region of OPLL, mm & \\
\hline
\end{tabular}

${ }^{*} p<0.05$ versus corresponding value at initial CT examination.

of ossification was as follows: continuous type, 17 subjects (15.6\%); segmental type, 82 (75.2\%); mixed type, 6 (5.5\%); and localized type, 4 (3.7\%). Furthermore, in 106 subjects (97.2\%) the cervical region had the maximum OPLL mass and in 3 subjects $(2.8 \%)$ the thoracic region had the maximum OPLL mass (Table 1). The mean follow-up period for CT was $80.8 \pm 15.5$ months (range 60-117 months). The thickness of the maximum region of OPLL, occupancy ratio of OPLL, and length of the maximum region of OPLL at initial CT examination were $3.4 \pm 1.0 \mathrm{~mm}, 28.8 \%$ $\pm 8.8 \%$, and $18.1 \pm 12.5 \mathrm{~mm}$, respectively. At the final CT examination, the respective measurements were $3.8 \pm 1.1$ $\mathrm{mm}, 31.4 \% \pm 9.8 \%$, and $20.2 \pm 15.2 \mathrm{~mm}$, respectively. The thickness of the maximum region of OPLL and occupancy ratio of OPLL at the final CT examination were significantly greater than that at the initial CT examination ( $\mathrm{p}<$ 0.05 ; Table 1). There were 3 subjects with suspected cancer (prostate, kidney, and lung) from the radiography interpretation report of the initial CT examination. Among these, 1 subject was diagnosed with renal cell carcinoma of the left kidney; this subject received a left nephrectomy to remove the cancer without any additional therapy, such as chemotherapy or radiation therapy.

\section{Comparison of Characteristics, OPLL Measurements, Laboratory Data, and BMD Status Between OPLL-NP and OPLL-P Groups}

Among the 109 subjects, 20 (18 men and 2 women) showed progression of ossification after more than 5 
TABLE 2. Comparison of OPLL measurements between the OPLL-NP $(n=89)$ and OPLL-P $(n=20)$ groups

\begin{tabular}{|c|c|c|c|}
\hline Variable & OPLL-NP & OPLL-P & $p$ Value \\
\hline Males, $\mathrm{n}(\%)$ & $73(82.0)$ & $18(90.0)$ & 0.517 \\
\hline Mean age (SD), yrs & $62.6(10.2)$ & $57.1(10.3)$ & 0.031 \\
\hline Mean BMI (SD), kg/m² & $24.9(2.9)$ & $25.6(3.9)$ & 0.387 \\
\hline Mean follow-up period of CT scans (SD), mos & $80.7(15.7)$ & $81.4(15.4)$ & 0.864 \\
\hline Diagnosis of cancer, $\mathrm{n}(\%)$ & $1(1.1)$ & $0(0.0)$ & 1.000 \\
\hline Cervical region of maximum OPLL mass, $\mathrm{n}(\%)$ & $86(96.6)$ & $20(100.0)$ & 1.000 \\
\hline OPLL $\geq 3$ vertebral levels, $n(\%)$ & $30(33.7)$ & $12(60.0)$ & 0.041 \\
\hline \multicolumn{4}{|l|}{ Type of OPLL, n (\%) } \\
\hline Continuous & $10(11.2)$ & $7(35.0)$ & 0.015 \\
\hline Segmental & $73(82.0)$ & $9(45.0)$ & 0.001 \\
\hline Mixed & $3(3.4)$ & $3(15.0)$ & 0.074 \\
\hline Localized & $3(3.4)$ & $1(5.0)$ & 0.561 \\
\hline \multicolumn{4}{|l|}{ OPLL size at initial examination (SD) } \\
\hline Thickness of maximum region of OPLL, mm & $3.4(1.0)$ & $3.6(1.2)$ & 0.405 \\
\hline Occupancy ratio of OPLL, \% & $28.3(8.5)$ & $30.9(9.9)$ & 0.234 \\
\hline Length of maximum region of OPLL, $\mathrm{mm}$ & $17.3(11.6)$ & $21.6(15.5)$ & 0.164 \\
\hline \multicolumn{4}{|l|}{ OPLL size at final examination (SD) } \\
\hline Thickness of maximum region of OPLL, mm & $3.6(1.1)$ & $4.4(1.3)$ & 0.003 \\
\hline Occupancy ratio of OPLL, \% & $29.8(9.1)$ & $38.3(9.9)$ & $<0.001$ \\
\hline Length of maximum region of OPLL, $\mathrm{mm}$ & $17.7(11.6)$ & $31.6(22.9)$ & $<0.001$ \\
\hline
\end{tabular}

years from baseline (OPLL-P group) and the incidence of OPLL progression was $18.3 \%$. The $\kappa$ value of interrater reliability for the judgment of OPLL progression was 0.941 , which indicates excellent reliability. There were no significant differences between the OPLL-NP and OPLL$\mathrm{P}$ groups with respect to sex, BMI, follow-up period of CT scan, diagnostic rate of cancer, or spinal region of maximum OPLL mass. The OPLL-P group had significantly younger age $(p=0.031)$ and higher prevalence of OPLL involvement of $\geq 3$ vertebral levels $(p=0.041)$ compared to the OPLL-NP group (Table 2). OPLL progression was significantly more common in patients with the continu-

TABLE 3. Comparison of initial data between the OPLL-NP $(n=$ $89)$ and OPLL-P $(n=20)$ groups

\begin{tabular}{lccc}
\hline \multicolumn{1}{c}{ Variable } & OPLL-NP & OPLL-P & p Value \\
\hline HbA1c, \% & $6.0(0.8)$ & $5.7(0.7)$ & 0.159 \\
\hline TG, mg/dl & $129.3(79.0)$ & $127.1(72.5)$ & 0.903 \\
\hline TC, mg/dl & $199.4(32.8)$ & $191.5(38.2)$ & 0.351 \\
\hline HDL cholesterol, mg/dl & $61.4(17.5)$ & $60.8(20.1)$ & 0.907 \\
\hline LDL cholesterol, mg/dl & $120.7(28.7)$ & $115.3(35.7)$ & 0.467 \\
\hline UA, mg/dl & $5.8(1.1)$ & $6.7(1.1)$ & 0.004 \\
\hline Calcium, mg/dl & $9.0(0.3)$ & $9.1(0.3)$ & 0.356 \\
\hline Phosphorus, mg/dl & $3.4(0.5)$ & $3.3(0.4)$ & 0.404 \\
\hline Bone quantitative ultrasound & & & \\
\hline \multicolumn{1}{c}{ BUA, dB/MHz } & $112.6(13.8)$ & $116.0(12.7)$ & 0.319 \\
\hline SOS, m/sec & $1557.8(33.7)$ & $1565.0(39.1)$ & 0.400 \\
\hline
\end{tabular}

Data are expressed as the mean (SD). ous type of OPLL ( $p=0.015$ ), whereas it was significantly less common in patients with the segmental type of OPLL $(\mathrm{p}=0.001$; Table 2). No significant between-groups differences were observed with respect to any OPLL measurements at initial CT examination. However, all OPLL measurements at final CT examination in the OPLL-P group were significantly greater than those in the OPLLNP group (Table 2).

Next, we compared the laboratory data and BMD status at the time of initial and final CT examinations between the two groups. The OPLL-P group showed significantly higher UA levels at the time of initial CT examination compared to the OPLL-NP group ( $\mathrm{p}=0.004$; Table 3), whereas UA levels at the time of final CT examination and other laboratory parameters at the time of initial and final CT examinations were comparable between the groups (Tables 3 and 4). With respect to the assessment of BMD status at the calcaneus using QUS, no significant between-groups differences were observed with respect to BUA or SOS at the time of initial and final CT examinations (Tables 3 and 4), indicating comparable BMD status of the calcaneus in both groups.

\section{Risk Factors for OPLL Progression}

Table 5 shows the results of the logistic regression analysis for OPLL progression after more than 5 years from baseline. Younger age (adjusted odds ratio [aOR] 0.95, 95\% CI 0.90-0.99), OPLL involvement of $\geq 3$ vertebral levels (aOR 2.88, 95\% CI 1.06-7.83), continuous type of OPLL (aOR 4.21, 95\% CI 1.35-13.10), and higher UA levels (aOR 2.09, 95\% CI 1.24-3.53) were significant risk factors for OPLL progression (Table 5). 
TABLE 4. Comparison of final data between the OPLL-NP and OPLL-P groups

\begin{tabular}{lccc}
\hline \multicolumn{1}{c}{ Variable } & OPLL-NP & OPLL-P & p Value \\
\hline $\mathrm{HbA1c}, \%$ & $6.1(0.8)$ & $5.9(0.6)$ & 0.177 \\
\hline $\mathrm{TG}, \mathrm{mg} / \mathrm{dl}$ & $123.5(80.7)$ & $117.9(58.2)$ & 0.772 \\
\hline $\mathrm{TC}, \mathrm{mg} / \mathrm{dl}$ & $187.9(27.3)$ & $181.3(27.5)$ & 0.330 \\
\hline $\mathrm{HDL}$ cholesterol, $\mathrm{mg} / \mathrm{dl}$ & $58.7(15.2)$ & $59.0(16.2)$ & 0.943 \\
\hline LDL cholesterol, mg/dl & $110.6(23.5)$ & $104.2(25.4)$ & 0.280 \\
\hline UA, mg/dl & $5.7(1.2)$ & $6.0(1.1)$ & 0.339 \\
\hline Calcium, mg/dl & $8.8(0.3)$ & $8.8(0.3)$ & 0.397 \\
\hline Phosphorus, mg/dl & $3.3(0.4)$ & $3.1(0.4)$ & 0.263 \\
\hline Bone quantitative ultrasound & & & \\
\hline BUA, dB/MHz & $125.2(106.7)$ & $116.2(11.7)$ & 0.709 \\
\hline SOS, m/sec & $1549.6(37.2)$ & $1560.6(34.1)$ & 0.229 \\
\hline
\end{tabular}

Data are expressed as the mean (SD).

\section{Illustrative Case}

A 65-year-old man was found to exhibit the mixed type of OPLL at the C2-6 levels on the sagittal view of a reconstructed CT scan (Fig. 2 left). The thickness of the maximum region of the OPLL, occupancy ratio of OPLL, and length of the maximum region of OPLL at initial CT examination were $6.1 \mathrm{~mm}, 45.5 \%$, and $45.9 \mathrm{~mm}$, respectively (Fig. 2 left). The ossified lesion had progressed both vertically (thickness of OPLL, $7.2 \mathrm{~mm}$; occupancy ratio of OPLL, 53.2\%) and horizontally (length of OPLL, 66.9 $\mathrm{mm}$ ) after 6 years from baseline (Fig. 2 right). The subject's UA level at the time of initial CT examination was $8.0 \mathrm{mg} / \mathrm{dl}$.

\section{Discussion}

To the best of our knowledge, this is the first observational study of OPLL progression in asymptomatic subjects based on long-term CT follow-up data (more than 5 years). Our study yielded two important findings: 1) the incidence of OPLL progression in asymptomatic subjects was $18.3 \%$ after at least 5 years from baseline, and 2) younger age, OPLL involvement of multiple vertebral levels, continuous type of OPLL, and higher UA levels were significant risk factors for progression of OPLL in asymptomatic subjects. These results may facilitate appropriate management of asymptomatic OPLL patients in clinical settings.

We evaluated OPLL progression by examining sagittal views of reconstructed CT scans, which is one of the strengths of our study. Measurements of the ossified le-

TABLE 5. Multivariable logistic regression analysis for OPLL progression

\begin{tabular}{lccc}
\hline \multicolumn{1}{c}{ Factor } & Adjusted OR & $95 \% \mathrm{Cl}$ & $\mathrm{p}$ Value \\
\hline Age & 0.95 & $0.90-0.99$ & 0.036 \\
\hline OPLL $\geq 3$ vertebral levels & 2.88 & $1.06-7.83$ & 0.038 \\
\hline Continuous type of OPLL & 4.21 & $1.35-13.10$ & 0.013 \\
\hline UA & 2.09 & $1.24-3.53$ & 0.006 \\
\hline
\end{tabular}

Adjusted for sex and BMI.
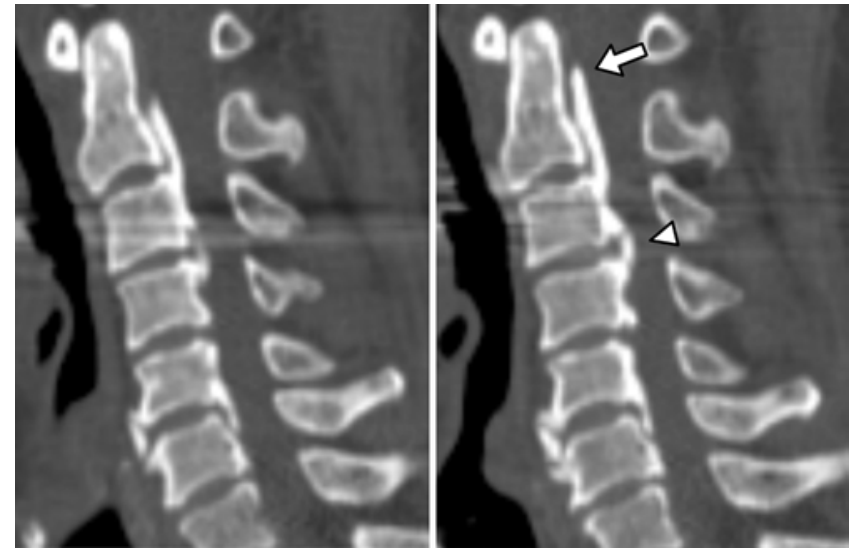

FIG. 2. Sagittal reconstructed CT images obtained in a 65 -year-old man with mixed type OPLL at the C2-6 levels at initial examination (left) and 6 years after initial examination (right). A CT image obtained 6 years after initial examination (right) showed a significant progression of the ossified mass both vertically (arrow) and horizontally (arrowhead).

sion using CT scans facilitate more precise identification of OPLL progression as compared to use of plain radiographs. Another strength of this study is the use of clinical data such as laboratory tests and BMD status (measured via QUS) to clarify the risk factors for OPLL progression.

Some previous studies have identified risk factors for OPLL progression in symptomatic patients with OPLL during the natural course of the disease. In a study by Katsumi et al., younger age was the most significant predictor of OPLL progression in nonsurgically treated patients; their study was based on 3D CT scans with a minimum follow-up period of 1 year. ${ }^{16}$ In a recent study by Park et al., young age, C2-3 involvement, and mixed type of OPLL were risk factors for OPLL progression in conservatively managed patients who underwent $\mathrm{CT}$ with a minimum follow-up of 2 years..$^{19}$ In our study, younger age, OPLL involvement of multiple vertebral levels, and continuous type of OPLL were identified as risk factors for progression of the ossified mass in asymptomatic subjects with OPLL. These results of asymptomatic subjects with OPLL are consistent with previous studies of symptomatic OPLL patients, including both in the postoperative course ${ }^{8-12,14,22}$ and in the natural course..$^{16,19}$

Our study also identified higher UA levels as a novel risk factor for OPLL progression in asymptomatic subjects with OPLL. Hyperuricemia is known to cause gouty arthritis due to deposition of urate crystals in tissues around the joints, tendons, and ligaments. ${ }^{25}$ The association between OPLL and hyperuricemia has not been reported. However, diffuse idiopathic skeletal hyperostosis, which is often accompanied by OPLL, ${ }^{6,26}$ was shown to be associated with hyperuricemia as well as several metabolic disorders (such as diabetes mellitus and hyperlipidemia). ${ }^{27,28}$ These previous findings may suggest a potential association between higher UA levels and hyperostosis in patients with OPLL. However, our results also showed that UA levels at the time of final CT examination were comparable between the groups (Table 4). One possible reason for the change in the statistical results of UA levels between initial and final examinations may be due to therapeutic inter- 
vention of the subjects who were identified as having hyperuricemia at initial examination. The final examination values of other laboratory parameters, such as TG, TC, or LDL cholesterol, were improved in both groups (Tables 3 and 4), which may indicate the effectiveness of therapeutic interventions for abnormal laboratory results at initial examination. There was no information regarding treatment or medication for hyperuricemia in this study. Therefore, further studies are required to determine the association between higher UA levels and progression of OPLL.

Several metabolic disorders, such as diabetes mellitus and obesity, were reportedly associated with OPLL. ${ }^{24}$ No significant differences were observed between the OPLLNP and OPLL-P groups with respect to BMI and HbA1c in our study (Tables 2-4). Given that we examined asymptomatic subjects who have a relatively small-sized OPLL mass (mean occupancy ratio of OPLL $=28.8$ ) in this study, there might not have been a significant correlation between metabolic disorders, such as diabetes mellitus and obesity, and OPLL progression.

Few studies have investigated the incidence of OPLL progression in the natural course using the most common criteria of progression (increase in ossified mass by $\geq 2 \mathrm{~mm}$ in thickness and/or length). Park et al. reported a $26.8 \%$ progression rate of vertical growth and $22.7 \%$ rate of thickness growth in the conservative course, which was defined by different cutoff values of progression (vertical progression was defined as $>2 \mathrm{~mm}$, but thickness progression was defined as $>1 \mathrm{~mm}) \cdot{ }^{19} \mathrm{In}$ our study, the incidence of OPLL progression in asymptomatic subjects was 18.3\% (20/109 subjects) based on the most commonly used criteria of OPLL progression; this is slightly lower than the rate reported in the previous study. The lower prevalence of OPLL progression in our study may be attributable to the difference in the definition of OPLL progression, as mentioned above, or to the relatively small size of ossification in asymptomatic subjects included in this study. Further large cohort studies using CT scans are needed.

Our study has several limitations. First, we did not randomly select healthy subjects, which may have introduced an element of selection bias. Second, we did not include other clinical information such as neurological dysfunction, bodily pain, or numbness. We confirmed that the study subjects did not have apparent clinical symptoms from medical interviews. However, subtle clinical symptoms or pyramidal signs (such as hyperreflexia) may have been unrecognized in our subjects. Moreover, we have not followed up on the study subjects and have not performed detailed clinical evaluation during the study period. Detailed clinical evaluation and follow-up information of study subjects may have provided more meaningful results. Third, we did not investigate the effect of segmental motion on progression of OPLL due to absence of dynamic radiographic examination. Fourth, we did not examine symptomatic patients with relatively large ossified masses. We expect to examine the risk factors such as higher UA levels in symptomatic OPLL patients in the future.

\section{Conclusions}

Our study demonstrated that progression of OPLL oc- curred in $18.3 \%$ of asymptomatic subjects at more than 5 years from baseline. Younger age, OPLL involvement of multiple vertebral levels, continuous type of OPLL, and higher UA levels were identified as significant risk factors for OPLL progression in asymptomatic subjects. A better understanding of the risk factors for progression of the ossified mass in asymptomatic subjects with OPLL will facilitate better clinical management of OPLL patients without symptoms or those with minor symptoms.

\section{Acknowledgments}

We thank all the orthopedic surgeons, their staff, and the patients who willingly participated in this study.

\section{References}

1. Fujimori T, Le H, Hu SS, et al. Ossification of the posterior longitudinal ligament of the cervical spine in 3161 patients: a CT-based study. Spine (Phila Pa 1976). 2015;40(7):E394E403.

2. Wang L, Jiang Y, Li M, Qi L. Postoperative progression of cervical ossification of posterior longitudinal ligament: a systematic review. World Neurosurg. 2019;126:593-600.

3. Matsunaga S, Sakou T. Ossification of the posterior longitudinal ligament of the cervical spine: etiology and natural history. Spine (Phila Pa 1976). 2012;37(5):E309-E314.

4. Onji Y, Akiyama H, Shimomura Y, et al. Posterior paravertebral ossification causing cervical myelopathy. A report of eighteen cases. J Bone Joint Surg Am. 1967;49(7):1314-1328.

5. Iwasaki M, Okuda S, Miyauchi A, et al. Surgical strategy for cervical myelopathy due to ossification of the posterior longitudinal ligament: Part 2: Advantages of anterior decompression and fusion over laminoplasty. Spine (Phila Pa 1976). 2007;32(6):654-660.

6. Fujimori T, Watabe T, Iwamoto Y, et al. Prevalence, concomitance, and distribution of ossification of the spinal ligaments: results of whole spine CT scans in 1500 Japanese patients. Spine (Phila Pa 1976). 2016;41(21):1668-1676.

7. Yonenobu K. Is surgery indicated for asymptomatic or mildly myelopathic patients with significant ossification of the posterior longitudinal ligament? Spine (Phila Pa 1976). 2012;37(5):E315-E317.

8. Chiba K, Yamamoto I, Hirabayashi H, et al. Multicenter study investigating the postoperative progression of ossification of the posterior longitudinal ligament in the cervical spine: a new computer-assisted measurement. J Neurosurg Spine. 2005;3(1):17-23.

9. Choi BW, Baek DH, Sheffler LC, Chang H. Analysis of progression of cervical OPLL using computerized tomography: typical sign of maturation of OPLL mass. J Neurosurg Spine. 2015;23(5):539-543.

10. Hori T, Kawaguchi Y, Kimura T. How does the ossification area of the posterior longitudinal ligament thicken following cervical laminoplasty? Spine (Phila Pa 1976). 2007:32(19):E551-E556.

11. Iwasaki M, Kawaguchi Y, Kimura T, Yonenobu K. Long-term results of expansive laminoplasty for ossification of the posterior longitudinal ligament of the cervical spine: more than 10 years follow up. J Neurosurg. 2002;96(2)(suppl):180-189.

12. Kawaguchi Y, Kanamori M, Ishihara H, et al. Progression of ossification of the posterior longitudinal ligament following en bloc cervical laminoplasty. J Bone Joint Surg Am. 2001;83(12):1798-1802.

13. Lee $\mathrm{CH}$, Sohn MJ, Lee $\mathrm{CH}$, et al. Are there differences in the progression of ossification of the posterior longitudinal ligament following laminoplasty versus fusion? A meta-analysis. Spine (Phila Pa 1976). 2017;42(12):887-894. 
14. Lee DH, Cho JH, Kim NH, et al. Radiological risk factors for progression of ossification of posterior longitudinal ligament following laminoplasty. Spine J. 2018;18(7):1116-1121.

15. Ogawa Y, Toyama Y, Chiba K, et al. Long-term results of expansive open-door laminoplasty for ossification of the posterior longitudinal ligament of the cervical spine. J Neurosurg Spine. 2004;1(2):168-174.

16. Katsumi K, Watanabe K, Izumi T, et al. Natural history of the ossification of cervical posterior longitudinal ligament: a three dimensional analysis. Int Orthop. 2018;42(4):835-842.

17. Matsunaga S, Sakou T, Hayashi K, et al. Trauma-induced myelopathy in patients with ossification of the posterior longitudinal ligament. J Neurosurg. 2002;97(2)(suppl):172-175.

18. Matsunaga S, Sakou T, Taketomi E, Komiya S. Clinical course of patients with ossification of the posterior longitudinal ligament: a minimum 10 -year cohort study. J Neurosurg. 2004;100(3)(Suppl Spine):245-248.

19. Park S, Lee DH, Ahn J, et al. How does ossification of posterior longitudinal ligament progress in conservatively managed patients? Spine (Phila Pa 1976). 2020;45(4):234-243.

20. Takatsu T, Ishida Y, Suzuki K, Inoue H. Radiological study of cervical ossification of the posterior longitudinal ligament. J Spinal Disord. 1999;12(3):271-273.

21. Yoshimura N, Nagata K, Muraki S, et al. Prevalence and progression of radiographic ossification of the posterior longitudinal ligament and associated factors in the Japanese population: a 3-year follow-up of the ROAD study. Osteoporos Int. 2014;25(3):1089-1098.

22. Tokuhashi Y, Ajiro Y, Umezawa N. A patient with two resurgeries for delayed myelopathy due to progression of ossification of the posterior longitudinal ligaments after cervical laminoplasty. Spine (Phila Pa 1976). 2009;34(2):E101-E105.

23. Tsuyama N. Ossification of the posterior longitudinal ligament of the spine. Clin Orthop Relat Res. 1984;(184):71-84.

24. Shingyouchi Y, Nagahama A, Niida M. Ligamentous ossification of the cervical spine in the late middle-aged Japanese men. Its relation to body mass index and glucose metabolism. Spine (Phila Pa 1976). 1996;21(21):2474-2478.
25. Perez-Ruiz F, Dalbeth N, Bardin T. A review of uric acid, crystal deposition disease, and gout. Adv Ther. 2015;32(1):3141.

26. Resnick D, Shapiro RF, Wiesner KB, et al. Diffuse idiopathic skeletal hyperostosis (DISH) [ankylosing hyperostosis of Forestier and Rotes-Querol]. Semin Arthritis Rheum. 1978;7(3):153-187.

27. Kiss C, Szilágyi M, Paksy A, Poór G. Risk factors for diffuse idiopathic skeletal hyperostosis: a case-control study. Rheumatology (Oxford). 2002;41(1):27-30.

28. Mader R, Verlaan JJ, Buskila D. Diffuse idiopathic skeletal hyperostosis: clinical features and pathogenic mechanisms. Nat Rev Rheumatol. 2013;9(12):741-750.

29. Sim J, Wright CC. The kappa statistic in reliability studies: use, interpretation, and sample size requirements. Phys Ther. 2005;85(3):257-268.

\section{Disclosures}

The authors report no conflict of interest concerning the materials or methods used in this study or the findings specified in this paper.

\section{Author Contributions}

Conception and design: Oshima, Doi. Acquisition of data: Doi, Sakamoto, Hirai. Analysis and interpretation of data: Oshima, Doi, Sakamoto. Drafting the article: Oshima, Doi. Critically revising the article: Oshima, Doi. Reviewed submitted version of manuscript: all authors. Approved the final version of the manuscript on behalf of all authors: Oshima. Statistical analysis: Doi, Horii. Study supervision: Oshima, Kato, Taniguchi, Matsubayashi, Hayashi, Tanaka.

\section{Correspondence}

Yasushi Oshima: University of Tokyo, Japan. yoo-tky@umin. ac.jp. 